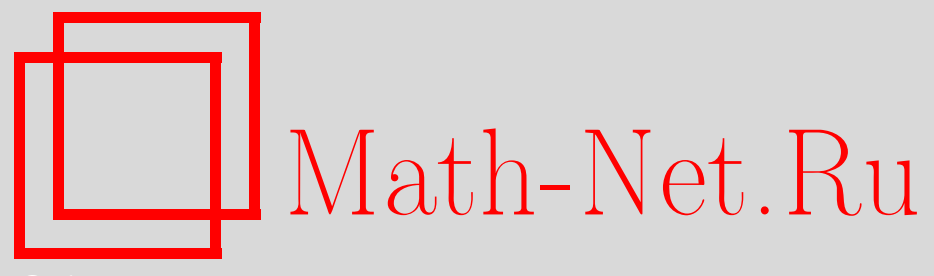

А. В. Мелешкина, Об абсолютной сходимости рядов Фурье по двукратным ограниченным полным ортонормированным системам, УМH, 2016, том 71, выпуск 2, 209210

DOI: https://doi.org/10.4213/rm9706

Использование Общероссийского математического портала Math-Net.Ru подразумевает, что вы прочитали и согласны с пользовательским соглашением http: //www . mathnet.ru/rus/agreement

Параметры загрузки:

IP : 34.227 .88 .159

26 апреля 2023 г., 12:04:13

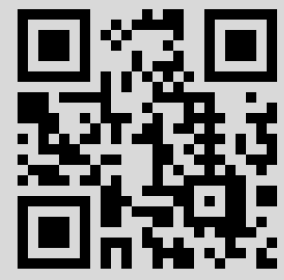




\section{Об абсолютной сходимости рядов Фурье по двукратным ограниченным полным ортонормированным системам}

\section{А. В. Мелешкина}

Пусть $\mathbb{I}^{n}=[0,1]^{n}-n$-мерный куб, $\mathbb{T}^{n}=[0,2 \pi)^{n}-n$-мерный тор и $C\left(\mathbb{I}^{n}\right), C\left(\mathbb{T}^{n}\right)$ пространства непрерывных на $\mathbb{I}^{n}$ и $\mathbb{T}^{n}$ функций. Модуль непрерывности функции $f \in C(X)$ для $X=\mathbb{I}^{n}, \mathbb{T}^{n}$ определяется равенством

$$
\omega(f, \delta)=\sup _{x, x+h \in X, 0<|h| \leqslant \delta}|f(x+h)-f(x)| .
$$

Пусть также $H^{\Omega}=\{f \in C(X): \omega(f, \delta) \leqslant \Omega(\delta), \delta>0\}$. А. Зигмундом установлено следующее достаточное условие абсолютной сходимости тригонометрического ряда Фурье функции ограниченной вариации (см. [1; гл. 4, § 4, с. 149]).

Теорема А. Если функиия $f(x) \in C\left(\mathbb{T}^{1}\right) \cap B V\left(\mathbb{T}^{1}\right)$ имеет модуль непрерывности, удовлетворяющий соотношению $\sum_{n=1}^{\infty} \sqrt{\omega(f, 1 / n)} / n<\infty$, то ряд из модулей коэфбичиентов Фурве по тригонометрической системе такой функции сходится.

Условие теоремы А для некоторых ортонормированных систем является слишком ограничительным. Например, ряд Фурье-Хаара каждой функции ограниченной вариации сходится абсолютно (см. [1; гл. 10, § 3, с. 420]). В [2] С. В. Бочкаревым доказано, что указанным свойством системы Хаара не может обладать ни одна равномерно ограниченная полная ортонормированная система функций. Точнее, им получена

Теорема В. Для любой равномерно ограниченной полной ортонормированной системы $\Phi=\left\{\varphi_{n}(x)\right\}_{n=1}^{\infty}$, определенной на $\mathbb{I}^{1}$, и модуля непрерывности $\Omega(\delta)$, для которого $\sum_{n=1}^{\infty} \sqrt{\Omega(1 / n)} / n=\infty$, найдется непрерьвная функция $F(x) \in H^{\Omega}$ с условием $F(0)=F(1)=0$, для которой расходится ряд из модулей коэфбициентов Фурве:

$$
\sum_{n=1}^{\infty}\left|c_{n}(F)\right|=\infty, \quad c_{n}(F)=\int_{0}^{1} F(x) \varphi_{n}(x) d x, \quad n=1,2, \ldots
$$

Из теоремы В вытекает существование функции $F(x) \in B V\left(\mathbb{I}^{1}\right)$ с модулем непрерывности $\omega(F, \delta)=O\left(\log ^{-2} \delta^{-1}\right), \delta \rightarrow 0$, для которой расходится ряд из модулей коэффициентов Фурье. Отметим также, что построение функций с абсолютно расходящимся рядом Фурье тесно связано с оценками снизу коэффициентов Фурье и $n$-членных приближений (см., например, [3]).

В данной заметке мы рассмотрим функции двух переменных $f(\mathbf{x})=f\left(x_{1}, x_{2}\right) \in$ $C(X)$, имеющие ограниченную вариацию по Харди, т.е. $f \in B V_{H}(X)$ (см. [4; рус. изд.: с. 768]). Для них также определим смешанный модуль непрерывности

$$
\omega\left(f, \delta_{1}, \delta_{2}\right)=\sup _{\mathbf{x}, \mathbf{x}+\mathbf{h} \in X, 0<h_{1} \leqslant \delta_{1}, 0<h_{2} \leqslant \delta_{2}}\left|\Delta_{\mathbf{h}}(f, \mathbf{x})\right|, \quad X=\mathbb{I}^{2}, \mathbb{T}^{2},
$$

где $\mathbf{h}=\left(h_{1}, h_{2}\right)$ и $\Delta_{\mathbf{h}}(f, \mathbf{x})=f\left(x_{1}, x_{2}\right)+f\left(x_{1}+h_{1}, x_{2}+h_{2}\right)-f\left(x_{1}+h_{1}, x_{2}\right)-f\left(x_{1}, x_{2}+h_{2}\right)$.

Аналогичный теореме А результат для функций из $B V_{H}\left(\mathbb{T}^{2}\right)$ и тригонометрической системы в двумерном случае вытекает из результатов работы А. Вереса [5] (см., например, следствие 2.6).

Теорема С. Если функиия $f(\mathbf{x}) \in C\left(\mathbb{T}^{2}\right) \cap B V_{H}\left(\mathbb{T}^{2}\right)$ имеет модуль непрерывности, удовлетворяющий соотношению

$$
\omega\left(f, \delta_{1}, \delta_{2}\right)=O\left(\log ^{-(2+\varepsilon)} \delta_{1}^{-1} \log ^{-(2+\varepsilon)} \delta_{2}^{-1}\right), \quad \delta_{1}, \delta_{2} \rightarrow 0,
$$

Исследование выполнено за счет гранта Российского научного фонда (проект № 14-50-00005).

DOI: $10.4213 / \mathrm{rm} 9706$ 
где $\varepsilon>0$, то ряд из модулей ее коэффициентов Фуръе по тригонометрической системе сходится.

Учитывая, что для функций вида $f(\mathbf{x})=f\left(x_{1}, x_{2}\right)=f_{1}\left(x_{1}\right) f_{2}\left(x_{2}\right)$ справедливо неравенство $\omega\left(f, \delta_{1}, \delta_{2}\right) \leqslant \omega\left(f_{1}, \delta_{1}\right) \cdot \omega\left(f_{2}, \delta_{2}\right)$ из теоремы В нетрудно вывести

СлЕДСТвИЕ. Для равномерно ограниченной полной ортонормированной системы вида $\Phi=\left\{\varphi_{n_{1}}\left(x_{1}\right) \varphi_{n_{2}}\left(x_{2}\right)\right\}_{n_{1}, n_{2}=1}^{\infty}$, определенной на $\mathbb{I}^{2}$, найдется непрерывная функизя $F(\mathbf{x}) \in B V_{H}\left(\mathbb{I}^{2}\right)$ с условием $F\left(x_{1}, 0\right)=F\left(x_{1}, 1\right)=F\left(0, x_{2}\right)=F\left(1, x_{2}\right)=0$ и моду лем непрерывности

$$
\omega\left(F, \delta_{1}, \delta_{2}\right)=O\left(\log ^{-2} \delta_{1}^{-1} \log ^{-2} \delta_{2}^{-1}\right), \quad \delta_{1}, \delta_{2} \rightarrow 0,
$$

для которой расходится ряд из модулей коэффициентов Фуръе.

Для систем общего вида такое следствие получить не удается. Однако справедлива

ТЕорема 1. Для любой равномерно ограниченной полной в $L^{2}\left(\mathbb{I}^{2}\right)$ ортонормированной системъ $\Phi=\left\{\varphi_{n}(\mathbf{x})\right\}_{n=1}^{\infty}$ найдется непрерывная функция $F(\mathbf{x}) \in B V_{H}\left(\mathbb{I}^{2}\right)$ с условием $F\left(x_{1}, 0\right)=F\left(x_{1}, 1\right)=F\left(0, x_{2}\right)=F\left(1, x_{2}\right)=0$ и модулем непрерывности

$$
\omega\left(F, \delta_{1}, \delta_{2}\right)=O\left(\log ^{-2} \frac{1}{\max \left\{\delta_{1}, \delta_{2}\right\}}\right), \quad \delta_{1}, \delta_{2} \rightarrow 0,
$$

для которой расходится ряд из модулей коэффичиентов Фуръе:

$$
\sum_{n=1}^{\infty}\left|c_{n}(F)\right|=\infty, \quad c_{n}(F)=\int_{0}^{1} \int_{0}^{1} F(\mathbf{x}) \varphi_{n}(\mathbf{x}) d \mathbf{x}, \quad n=1,2, \ldots
$$

Доказательство теоремы 1 основано на построениях из [1; теорема 10.11]. Мы используем следующий вспомогательный результат, являющийся модификацией неравенства типа Гальярдо-Ниренберга.

Лемма. Для функиии $f(\mathbf{x})$, определенной на $(0, a)^{2}$ и удовлетворяющей условию $\left\|\partial^{2} f / \partial x_{1} \partial x_{2}\right\|_{L^{\infty}(0, a)^{2}} \leqslant 1$, и любого числа $\beta, 0<\beta<\min \{1, a\}$, имеет место неравенство

$$
\begin{aligned}
& \int_{\beta}^{a} \int_{\beta}^{a} h_{1}^{-2} h_{2}^{-2} \int_{0}^{a-h} \int_{0}^{a-h}\left(\Delta_{\mathbf{h}}(f, \mathbf{x})\right)^{2} d \mathbf{x} d \mathbf{h} \\
& \quad \leqslant 32\left[\beta^{2} \int_{0}^{a} \int_{0}^{a}|f(\mathbf{x})| d \mathbf{x}+\left(\log \frac{a}{\beta}\right) \iint_{\left\{\mathbf{x} \in(0, a)^{2}:|f(\mathbf{x})|>\beta^{4}\right\}}|f(\mathbf{x})| d \mathbf{x}\right] .
\end{aligned}
$$

Вопрос о точности теоремы 1 остается открытым и тесно связан с открытым вопросом Б. С. Кашина о справедливости двумерного аналога неравенства (8) из [6] (с заменой в двумерном случае обычной производной на смешанную производную $\left.\partial^{2} f / \partial x_{1} \partial x_{2}\right)$.

\section{Список литературы}

[1] Б. С. Кашин, А.А. Саакян, Ортогоналънъе рядъ, 2-е изд., АФЦ, М., 1999, х +550 с. [2] С. В. Бочкарев, УМH, 27:2(164) (1972), 55-81. [3] А. В. Мелешкина, Матем. заметки, 97:4 (2015), 632-635. [4] Математическая энциклопедия, т. 5, ред. И. М. Виноградов, Советская энциклопедия, М., 1985, 1248 с. [5] A. Veres, Acta Sci. Math. (Szeged), 74:1-2 (2008), 191-206. [6] Б. С. Кашин, Матем. сб., 106(148):3(7) (1978), 380-385.

А. В. Мелешкина (А. V. Meleshkina)

Математический институт им. В. А. Стеклова

Российской академии наук

E-mail: meleshkina-anna@mail.ru
Представлено А. Г. Сергеевым Принято редколлегией 24.02.2016 